\title{
Future Industrial Kitchen: Challenges and Opportunities
}

\author{
Lucas Pereira \\ ITI, LARSyS, Técnico Lisboa \\ Lisbon, Portugal \\ lucas.pereira@tecnico.ulisboa.pt
}

\author{
Vitor Aguiar \\ ITI, LARSyS, M-ITI \\ Funchal, Portugal \\ vitor.aguiar@m-iti.org
}

\author{
Fábio Vasconcelos \\ ITI, LARSyS, M-ITI \\ Funchal, Portugal \\ fabio.vasconcelos@m-iti.org
}

\begin{abstract}
Large amounts of electricity, water, and food are used every day in Industrial Kitchens (IK). Still, very little attention has been devoted by the research community to this potential source of resource over-consumption. This abstract paper builds on the deployment of sensing technology in three IKs to present the main challenges and potential research directions towards more sustainable IKs.
\end{abstract}

\section{CCS CONCEPTS}

- Social and professional topics $\rightarrow$ Sustainability; • Computing methodologies $\rightarrow$ Machine learning approaches.

\section{KEYWORDS}

electricity, water, waste, industrial kitchen, sustainability

\section{INTRODUCTION}

Industrial Kitchens are among the highest consumers of electricity and water [1,2], as well as of waste production. Still, very little attention has been devoted to this area by the research community. The Future Industrial Kitchen (FIK) project aims at understanding the interactions between electricity, water, and waste in high-end restaurants. To this end, electricity, water, and waste monitoring technology have been in three restaurants for consecutive periods of 4 weeks (see Figure 1). This abstract paper presents the main challenges encountered during the deployments and outlines potential research directions.

\section{ELECTRICITY MONITORING}

Since almost all of the electric appliances appear directly connected to the main breaker box, it is not possible to use plug-level smart-meters. For this reason, the FIK research team resorted to the eGauge Pro ${ }^{1}$ circuit-level meter. More specifically, 30 individual circuits have been monitored in each IK, including loads such as refrigerators, convection ovens, and salamanders. A Fluke 438- $\mathrm{II}^{2}$ meter was also used to monitor the aggregate consumption at each of the three phases.

\footnotetext{
${ }_{1}^{1}$ eGauge Pro, https://www.egauge.net/eos/energy-meters/EG4130

${ }^{2}$ Fluke 438-II, https://www.fluke.com/en/product/electrical-testing/power-qualityanalyzers/fluke-438-ii

Permission to make digital or hard copies of all or part of this work for personal or classroom use is granted without fee provided that copies are not made or distributed for profit or commercial advantage and that copies bear this notice and the full citation on the first page. Copyrights for components of this work owned by others than ACM must be honored. Abstracting with credit is permitted. To copy otherwise, or republish, to post on servers or to redistribute to lists, requires prior specific permission and/or a fee. Request permissions from permissions@acm.org.

BuildSys '19, November 13-14, 2019, New York, NY, USA

(c) 2019 Association for Computing Machinery.

ACM ISBN 978-1-4503-7005-9/19/11 . \$ \$15.00

https://doi.org/10.1145/3360322.3360872
}

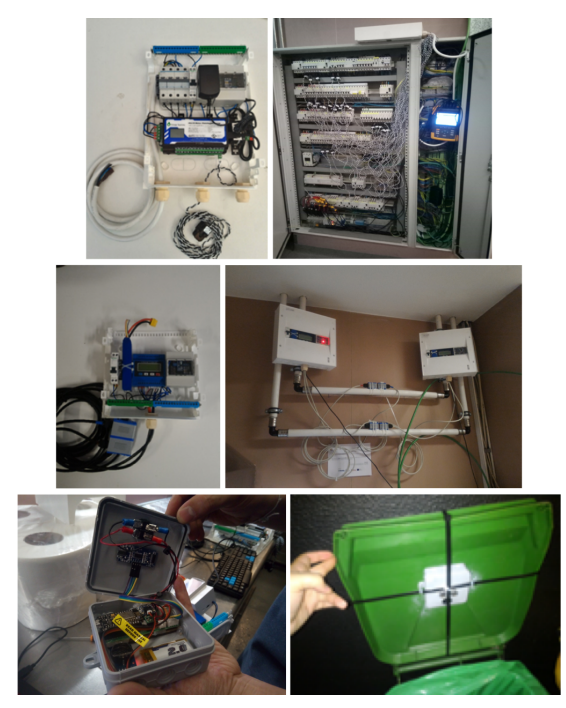

Figure 1: Sensing hardrware deployed: electricity (top), water (centre), waste (bottom).

The first aspect that was noticed was the low energy explained in any of the three IKs (50\%, 66\%, and $40 \%$ respectively) despite the high number of monitored circuits.

As the IoT becomes ubiquitous, appliances may return their consumption in real-time. Nevertheless, the benefits from real-time monitoring of the individual loads go beyond consumption. One of the potentialities of such data is the possibility of detecting malfunctioning appliances. As such, Non-Intrusive Load Monitoring (NILM) has a role in the context of IK. One of the limitations of NILM is that it does not enable load actuation without additional hardware. Thus, IKs call for hybrid solutions, where NILM is used to monitor always-on loads / non-deferrable loads, and IoT sensors to enable the actuation in specific loads.

It was also noticed that the dynamic nature of IKs operation leads to considerable phase unbalance in the electric installation (see Figure 2). Such an unbalance can ultimately lead to quicker degradation of the loads.

With the advent of Battery Energy Storage Systems (BESS), it should be possible to use such devices to balance the phases, by controlling the injection of active and reactive power in each of the grid phases. Furthermore, BESS can also be used to perform arbitrage, e.g., charge with energy from periods with more renewable energy on the grid, and use it to cover peaks in consumption. In fact, from Figure 2, it is possible to observe that the highest consumption happens early in the night, where electricity usually is more expensive to get. 


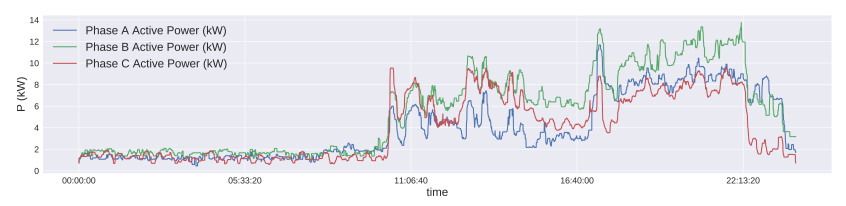

Figure 2: Average active power per phase during 24 hours of operation on a selected IK.

\section{WATER MONITORING}

In the context of IK operations, water serves two main purposes, cooking and cleaning (e.g., dish and glass washing), where the second is by far more water-intensive.

Just like the case of electricity, it is not feasible to monitor each water fixture individually. Thus the alternative is to use nonintrusive water monitoring. In this case, the TUF2000M ${ }^{3}$ ultrasonic flow sensor was used to monitor hot and cold water.

Since the number of appliances that use water is low, it should be fairly straightforward to disaggregate individual consumption, especially by combining water and electricity data streams. Nevertheless, a more exciting feature of high-frequency water monitoring is the possibility of identifying malfunctioning loads and problems in the water distribution system.

\section{WASTE MONITORING}

IKs produce considerable amounts of waste where the more prominent are undifferentiated, paper, plastic, and glass. Waste generation happens in two stages, the food preparation, and during service. In the scope of IK, one of the critical features of waste monitoring is the ability to keep track of how waste is produced and inform when to clean waste bins. Nevertheless, when integrated with other information systems like stock management, it should be possible to monitor the actual waste of food.

In the FIK project, ultra-sonic distance sensors ${ }^{4}$ were used to monitor the volume of the waste in individual bins. However, the results in practice were far from those expected in theory. This happened in part to the harsh conditions of operation and the difficulty to get correct measurements due to the inconsistent operation of the garbage bins across IKs.

One alternative is to use image recognition algorithms to calculate the volume of waste [3]. However, at this stage, this solution is rather expensive and not easily applicable to smaller bins like the ones found inside IKs. Thus, to monitor waste generation near real-time, a combination of sensors should be used. For example, more than one distance sensor, a scale, and other IoT sensors to identify when the lead opens, and when bins move from one place to another. Furthermore, it should also be possible to identify the waste in each bin. This is particularly relevant for waste to energy research, where the presence of "unwanted" materials in the mix can lead to considerable inefficiencies. Finally, all these sensors should require very little power to work, due to the almost certain inability to rely on power from the grid.

\footnotetext{
${ }^{3}$ TUF: Ultrasonic Water Flow Meter, http://www.t3-1.com/english/index.php ${ }^{4}$ HC-SR04 Range Finder, https://www.acmesystems.it/HC-SR04
}

\section{OTHER CONSIDERATIONS}

Gas Monitoring. With the advent of electric stoves and burners, the usage of gas in IKs is now less common. However, this is something that should be taken into consideration by the research community. The FIK project did not consider gas, but any monitoring solution will have to be non-intrusive, possibly using ultra-sonic gas flow meters ${ }^{5}$.

Temperature and Humidity. One of the critical aspects of IKs is to keep the temperatures and humidity within the correct thresholds. Thus HVAC of crucial importance, and one of the topics currently being tackled by the IK industry ${ }^{6}$. Nevertheless, the price of exiting solutions are unpractical to most IK owners. Thus it would be very relevant to have the ability to perform this operation at lowers costs. This would necessarily, imply the deployment of low cost/low power sensing to control the HVAC systems dynamically.

Human Activity Detection and Classification. Our research has shown that in the scope of IKs, each operator has a particular task, and stays most of the time in the same location. Nevertheless, being able to detect and classify human activity would be highly relevant when it concerns kitchen automation. For example, by identifying long periods of absence of a particular staff member, it would be possible to detect resource over-consumption (e.g., water or electricity), and act accordingly. There is already an extensive body of research in human tracking, where Ultra Wide Band (UWB) sensing is one of the most attractive options. However, after experimenting with this technology, it was noted that the physical conditions of IKs are far from ideal for this kind of operation, mainly due to the presence of highly reflective materials. Thus, research in this direction should consider sensing techniques that require minimal hardware installation.

Benchmark Metrics. Literature [1,2] reveals that the benchmark metrics most commonly employed in IKs are ratios between energy consumption and IK characteristics like size, seats, and meals served. However, additional metrics are needed, in particular metrics that provide estimates of the level of quality of the IK equipment, for example by comparing the ideal operation, operation in the past and current operation of appliances it would be possible to provide the IK operators with instant snapshots of the kitchen operation.

\section{ACKNOWLEDGMENTS}

This research was funded by projects M1420-01-0247-FEDER-000018 (Madeira 14-20), and UID/EEA/50009/2019 (FCT).

\section{REFERENCES}

[1] Etienne Paillat. 2011. Energy Efficiency In Food-Service Facilities: The Case Of Långbro Värdshus Etienne Paillat. Master's thesis. KTH Royal Institute of Technology, Stockholm, Sweden.

[2] R. Hedrick, V. Smith, and K. Field. 2011. Restaurant Energy Use Benchmarking Guideline. Technical Report NREL/SR-5500-50547, 1019165. National Renewable Energy Laboratory. https://doi.org/10.2172/1019165

[3] Kazuhiro Mikami, Yin Chen, and Jin Nakazawa. 2018. Using Deep Learning to Count Garbage Bags. In Proceedings of the 16th ACM Conference on Embedded Networked Sensor Systems (SenSys '18). ACM, Shenzhen, China, 329-330. https: //doi.org/10.1145/3274783.3275167

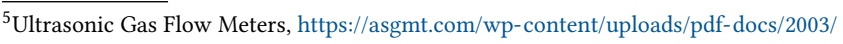
1/11.pdf

${ }^{6}$ Intellihood, https://melinkcorp.com/intellihood
} 